\title{
The effect of prior antimicrobial therapy for community acquired infections on the aetiology of early and late onset ventilator-associated pneumonia in a level I trauma intensive care unit
}

\author{
Yogandree Ramsamyab*, David JJ Muckartc, Khine Swe Swe Han ${ }^{b}$ and Koleka P Mlisana ${ }^{b}$ (D) \\ ${ }^{a}$ Department of Microbiology, Prince Mshiyeni Memorial Hospital, Durban, South Africa \\ ${ }^{b}$ Department of Medical Microbiology, School of Laboratory Medicine and Medical Sciences, University of KwaZulu-Natal, National Health \\ Laboratory Services (KZN Academic Complex), Durban, South Africa \\ 'Department of Surgery, Nelson R Mandela School of Medicine, University of KwaZulu-Natal, Durban, South Africa \\ *Corresponding author, emails:yogandree@gmail.com, Ramsamyy@ukzn.ac.za

Background: Ventilator-associated pneumonia (VAP) is the most common hospital acquired infection in patients who require mechanical ventilation. Early VAP is associated with community acquired pathogens whereas late VAP involves hospital flora. Based on this premise, a protocol may be formulated for microbiological surveillance and antimicrobial stewardship within a specific intensive care unit (ICU) to ensure appropriate empiric antimicrobial choice. The bacterial flora in VAP may be affected, however, by antimicrobials prescribed during the ICU stay.

Aim: The aim of this study was to determine the effect of prior antimicrobial therapy for community acquired infections on aetiology and the susceptibility of bacterial isolates from the first episode of early or late VAP in a trauma intensive care unit. Methods: Endotracheal aspirates (ETAs) were obtained from patients with suspected early and late VAP. All ETAs were processed and interpreted as per the Clinical and Laboratory Standards Institute (CLSI). Patients were divided into two cohorts: those whose injuries had required antimicrobial therapy for community acquired infections and those who were antimicrobial naïve. The effect of prior antimicrobial therapy on bacterial isolates from the first episode of suspected VAP was compared between the two groups.

Results: Of 288 patients admitted to the Trauma ICU between January and December 2014, pneumonia was suspected in 91 (31.6\%). Of these, 69 (76\%) patients were antimicrobial naïve and $22(24 \%)$ had received prior antimicrobial therapy. Early VAP occurred in $31(45 \%)$ patients in the naïve cohort compared to $3(12.5 \%)$ with prior antimicrobial exposure $(p=0.01)$. Of the early VAP isolates $25(81 \%)$ in the naïve cohort contained community flora, whereas all isolates in those with prior antimicrobial therapy revealed hospital acquired organisms $(p=0.01)$. In the antimicrobial naïve cohort with late VAP $27(71 \%)$ patients had community acquired organisms, whereas only $3(16 \%)$ isolates in late VAP in those with prior therapy revealed community acquired flora $(p<0.001)$.

Conclusion: Patients who receive prior antimicrobial therapy have a significantly lower incidence of early VAP, but in those who developed either early or late VAP hospital acquired pathogens were more commonly isolated. Knowledge of prior antimicrobial exposure in a patient with early or late VAP will assist in determining the correct empiric antimicrobial choice.

\section{Introduction}

Ventilator-associated pneumonia (VAP) as defined by the Centre for Disease Control (CDC) is pneumonia occurring after $48 \mathrm{~h}$ of endotracheal intubation and mechanical ventilation. ${ }^{1}$ Early VAP occurs on the third and fourth days and late from day five onwards. Although direct attributable mortality is difficult to estimate $^{2}$ most studies report an association with prolonged mechanical ventilation, hospital stay and increased cost. ${ }^{3}$ The empiric choice of antimicrobial agents is an important determinant of morbidity 4,5 and is guided by the time of onset of VAP and microbiological surveillance for that ICU. ${ }^{6}$

Indiscriminate antimicrobial use has promoted the emergence of multi-drug resistant organisms and stewardship and surveillance supports prescribing appropriate antimicrobials with the narrowest spectrum of activity to cover the most likely pathogens. ${ }^{7}$ Early VAP has a strong association with susceptible community acquired organisms whereas more resistant pathogens are isolated almost invariably in late VAP as described by Gastmeier et al. ${ }^{8}$ Early-onset nosocomial pneumonias were due primarily to Gram-negative bacteria, such as Haemophilus influenzae, and Gram-positive methicillin sensitive Staphylococcus aureus (MSSA) and Streptococcus pneumonia. Other bacterial causes include Moraxella catarrhalis, Klebsiella pneumoniae, and atypical CAP pathogens such as Chlamydia pneumoniae, Mycoplasma pneumoniae and Legionella pneumophila. ${ }^{9}$ For lateonset nosocomial pneumonia, the most commonly encountered causative pathogens reported were antibiotic-resistant Gramnegative bacteria, such as Pseudomonas aeruginosa, Acinetobacter spp., or methicillin-resistant S. aureus (MRSA). The most prevalent pathogens, causing $80 \%$ of hospital acquired pneumonia, are S. aureus, P. aeruginosa, Klebsiella spp., E. coli, Acinetobacter spp., and Enterobacter spp..$^{10}$

Prior antimicrobial exposure for community acquired infections may affect the sensitivity patterns of bacterial isolates in both early and late health care associated infections. This information is essential as it may influence the choice of empiric antimicrobials. To ascertain the effect of such therapy on the time of onset and sensitivity of bacterial isolates in VAP, we undertook a prospective observational study in a trauma intensive care unit. 


\section{Patients and methods}

This study was performed from 1 January to 31 December 2014 in the Trauma Intensive Care Unit (TICU) at the Inkosi Albert Luthuli Central Hospital (IALCH). IALCH is a tertiary/quaternary public service institution in Durban, South Africa. The TICU at IALCH is exclusively for critically injured patients requiring mechanical ventilation. The unit provides ventilatory support for adults and children and all patients were included regardless of age. The patient cohort was used in a previously published study by Ramsamy et al. ${ }^{11}$ in 2016.

Patients who underwent mechanical ventilation in the TICU from 1 January to 31 December 2014 were identified using prospectively collected data gathered from the TICU computerised database. VAP was defined as pneumonia occurring in a patient after $48 \mathrm{~h}$ from the time of intubation with an endotracheal or tracheostomy tube and which was not present before admission. ' To reduce the incidence of VAP, a 'VAP bundle' is used routinely in the TICU. This includes strict handwashing, head elevation to between $30-45$ degrees unless contraindicated, oral care using chlorhexidine, endotracheal tubes with a subglottic aspiration port, early enteral feeding, stress ulcer prophylaxis if enteral feeding is delayed, deep venous thrombosis (DVT) prophylaxis, minimising sedation, and early trials of spontaneous ventilation with a view to extubation as soon as possible. ${ }^{12}$ The diagnostic criteria for VAP as per CDC were the combination of new onset pyrexia $>38.0$ degrees Celsius, changes on chest auscultation, new infiltrates on chest radiology, purulent endotracheal aspirate (ETA) and a rise or fall in white cell count coupled with a deterioration in lung function manifested by a reduction in the $\mathrm{PaO}_{2} / \mathrm{FiO}_{2}$ ratio and reduced compliance. 'Patients with no record of ETA's, those who did not fulfil the CDC definitions, and those previously treated for other nosocomial infections were excluded. All specimens were processed at the National Health Laboratory Services (NHLS) situated at IALCH. All data relevant to the specimens collected from all the study patients were obtained from the laboratory computer database. Microbiological processing of ETA's, pathogen identification and antimicrobial susceptibility testing was carried out as per standard operating procedure of the NHLS and Clinical and Laboratory Standards Institute. ${ }^{13}$ Quantitative cultures of ETA are not routinely performed. The quality of every ETA sample is assessed using the Barlett scoring system. ${ }^{14}$ Microscopy and culture results of each ETA are correlated with the clinical condition of each patient. The purpose of this is to differentiate possible infection and colonisation. Pathogen identification and antimicrobial susceptibility testing was carried out using the Vitek $2^{\circledR}$ (BioMerieux, Midrand, South Africa) platform. All laboratory results were correlated with the patients' clinical condition. Based on previous microbiological surveillance the empiric antimicrobial policy for the TICU is amoxycillin/ clavulanic acid for early-onset VAP and piperacillin/tazobactam for late-onset VAP. Combination therapy is not employed.

Following abdominal trauma with a breach of the gastrointestinal tract patients are routinely commenced on empiric antimicrobial therapy for secondary peritonitis with community acquired faecal organisms. Our empiric antimicrobial of choice is amoxycillin/clavulanic acid. Patients with contaminated compound fractures or contaminated soft tissue injury are treated in a similar manner, although the choice of antimicrobial will depend on the degree of contamination and likely pathogens.
Our policy for prophylactic antimicrobials is to use a single dose of cefazolin for orthopaedic internal fixation and cefoxitin for abdominal surgery.

Patients were divided into those who had been prescribed antimicrobials for their injury and those who were antimicrobial naive at the time of the first episode of suspected VAP. Those receiving antimicrobial prophylaxis for $24 \mathrm{~h}$ or less were considered antimicrobial naïve. The incidence of early and late VAP, the organisms isolated and their susceptibility were compared between the groups. Chi squared or Fisher's exact test was used to determine significance at a level of $p<0.05$. This study was approved by the Bioethics Committee of the University of KwaZulu-Natal (BE 207/09).

\section{Results}

During the study period a total of 288 patients were admitted to the TICU, of whom 106 (36.8\%) were suspected of developing VAP. There were 84 (79.2\%) males and 22 (20.8\%) females with a median age of 29 years (IQR 21 - 37) and a median Injury Severity Score (ISS) of 31 (IQR 24 - 38). Mechanism of injury was predominantly blunt, with $77(72.6 \%)$ having been injured in motor vehicle collisions, 17 (16.1\%) in non-vehicular blunt trauma, $8(7.5 \%)$ following gunshot wounds, $3(2.8 \%)$ stab wounds, and $1(1 \%)$ snake bite. Fifteen patients were excluded from analysis. Pneumonia was suspected in 8 patients $<48$ hours after intubation and mechanical ventilation, respiratory flora were isolated after suspected aspiration in 3 patients, and Candida spp. was the sole isolate in 2 patients. These did not conform to the CDC definitions of VAP. In the remaining 2 patients, prior antimicrobials had been administered for other mechanisms of nosocomial sepsis. This left a cohort of 91 (31.6\%) patients. Of these, $69(75.8 \%)$ patients were antimicrobial naïve and $22(24.2 \%)$ had received prior antimicrobial therapy, consisting of amoxycillin/clavulanic acid in 17 (77.3\%), cloxacillin in $3(13.6 \%)$ and cefazolin in the remaining $2(9.1 \%)$ patients. Early VAP occurred in 34 (37.4\%) and late VAP in 57 (62.6\%) patients. The frequency of early and late VAP and whether bacterial isolates were community or hospital acquired pathogens are illustrated in Table 1.

In the antibiotic naïve cohort, there were similar numbers of early and late onset VAP. There were more cases of late onset VAP in the patients who received prior antimicrobials. Thirty-one (44.9\%) patients developed early VAP compared to $3(13.6 \%)$ in those with prior therapy $(p=0.01 ; \mathrm{OR}=5.16 ; 95 \% \mathrm{Cl} 1.4-19.1)$. In the antibiotic naïve group community acquired pathogens

Table 1: Number of patients with early and late VAP and community versus hospital acquired pathogens for the first episode of VAP in antimicrobial naïve patients and those receiving prior therapy

\begin{tabular}{|l|c|c|}
\hline & Early VAP & Late VAP \\
\hline No Antibiotics & & \\
\hline Community pathogens & 25 & 27 \\
\hline Hospital pathogens & 6 & 11 \\
\hline Total & 31 & 38 \\
\hline Antibiotics & & \\
\hline Community pathogens & 0 & 3 \\
\hline Hospital pathogens & 3 & 16 \\
\hline Total & 3 & 19 \\
\hline
\end{tabular}


predominated in both early and late onset VAP. Community acquired organisms were isolated in $25(80.6 \%)$ patients in the naïve group but, in all $3(100 \%)$ episodes of early VAP in those with prior therapy, hospital acquired pathogens were isolated $(p=0.01 ; \mathrm{OR}=27.5 ; 95 \% \mathrm{Cl} 1.2-600.0)$. Of the 38 patients in the naïve cohort who developed late onset VAP, bacterial isolates revealed community acquired pathogens in $27(71 \%)$. In patients who received prior antimicrobial therapy, hospital acquired pathogens were more commonly isolated. In the 19 patients with prior therapy, only $3(15.8 \%)$ isolates contained community acquired organisms $(p<0.001 ; \mathrm{OR}=13.16 ; 95 \% \mathrm{Cl} 3.17-54.1)$

In our patient population who were antimicrobial naïve, the pathogens isolated in early VAP were similar to published research. Of interest was the finding that in this patient cohort $H$. influenzae sensitive to amoxycillin-clavulanic acid and methicillin-susceptible $S$. aureus remained the most common isolates in suspected late VAP. In addition, almost $90 \%$ of Klebsiella pneumoniae isolates in late VAP maintained susceptibility to amoxycillin-clavulanic acid. Given the fact that almost $80 \%$ of patients who had received prior antimicrobial therapy had been treated with amoxycillin-clavulanic acid, the virtual absence of early VAP and community flora in both early and late VAP is not surprising. A. baumannii, E. cloacae and $P$. aeruginosa accounted for almost $80 \%$ of isolates in this cohort. These organisms are uncommon in early VAP and would normally not have been susceptible to the recommended empiric therapy with amoxicillin-clavulanic acid for community acquired pneumonia.

In addition to the 15 patients excluded from analysis, 19 patients died within $12 \mathrm{~h}$ of admission due to devastating injury and therefore did not survive long enough to develop VAP. This left a total of 163 patients who survived more than $48 \mathrm{~h}$ without acquiring VAP. Fourteen (15.4\%) of the 91 patients with VAP died compared to $18(11.0 \%)$ of the 163 without VAP $(p=0.33$; OR $=$ $1.46 ; 95 \% \mathrm{Cl} 0.69-3.10)$. If the 15 exclusions are included in the non-VAP cohort the result remains non-significant ( $p=0.23$; OR $=$ $1.6 ; 95 \% \mathrm{Cl} 0.76-3.42)$. There were $4(11.8 \%)$ deaths in the 34 patients with early VAP and $10(17.5 \%)$ deaths in the 57 patients with late onset VAP $(p=0.56 ; \mathrm{OR}=0.63: 95 \% \mathrm{Cl} 0.18$ - 2.18).

The distribution of pathogens in early and late VAP in the antibiotic naïve and prior antibiotic groups is illustrated in Figures 1 and 2, respectively.

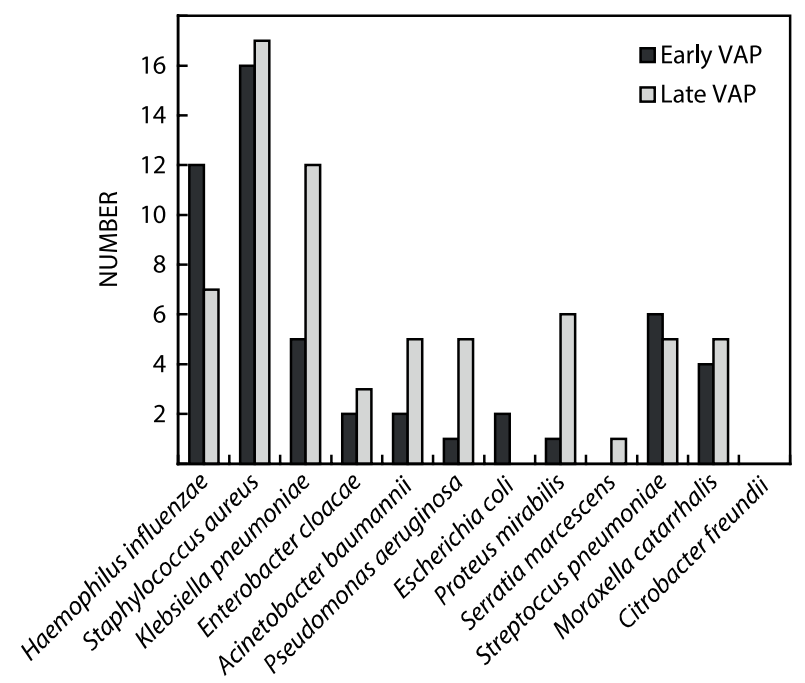

Figure 1: Distribution of pathogens in early and late VAP in antimicrobial naïve patients.

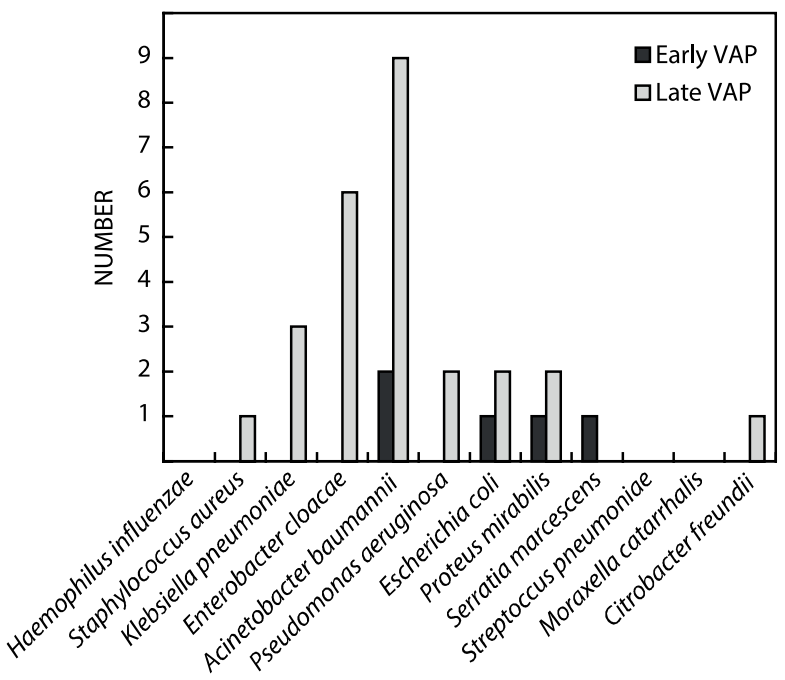

Figure 2: Distribution of pathogens in early and late VAP in patients receiving prior antimicrobial therapy.

\section{Discussion}

Pneumonia is the most common hospital acquired infection in patients who require mechanical ventilation occurring at a rate of $1 \%-3 \%$ per day. ${ }^{15}$ Although a direct association with mortality has been difficult to prove, VAP increases the duration of mechanical ventilation, length of ICU stay and hospital costs. ${ }^{4}$ Should VAP occur, the timely administration of appropriate empirical antimicrobial therapy has been shown to have a major impact on such morbidity. ${ }^{16}$ Early-onset VAP is most often due to community acquired pathogens, whereas the majority of late onset VAP's are caused by antimicrobial-resistant Gram-negative organisms. The incidence of methicillin resistant $S$. aureus is variable but is uncommon in our patient population, the most likely explanation being a low incidence of prior antimicrobial use in the community. Hence guidelines for empiric therapy for early onset infection recommend monotherapy with narrowspectrum antimicrobials for early-onset infections and agents with a broader spectrum for late-onset infections. ${ }^{17-19}$

The pathogens isolated in our study are similar to those previously reported for VAP in the critically injured..$^{20}$ The Gramnegative Enterobacteriaceae were the most common pathogens isolated, especially in late VAP. Amongst the Gram-positive species which were less common, $S$. aureus was the most commonly isolated pathogen. Extended spectrum beta lactamase producing organisms and MRSA were amongst the minority of the pathogens isolated for which there are a number of explanations. The TICU employs a strict infection control policy in addition to a policy of antimicrobial stewardship and antimicrobial prescribing that is based on microbiological surveillance. Furthermore, patients treated in the TICU are a fairly unique population compared to those admitted to general medical and surgical intensive care units. The TICU is exclusively for critically injured patients who require mechanical ventilation. This is an otherwise well population free from chronic medical conditions, previous hospital visits or the prior use of antimicrobials. Although the isolated flora may be similar in intensive care units the extent and specifics of bacterial resistance to antimicrobials varies considerably, the most common reasons are prior antimicrobial exposure, the overuse of broad spectrum agents and selective pressure. ${ }^{11}$ Despite suggestions that the differentiation between early and late VAP is irrelevant and that all patients should be treated with broad spectrum agents ${ }^{16}$ our 
data confirm that this distinction remains useful when deciding upon empiric therapy. ${ }^{11}$ In our study it was observed that community acquired flora susceptible to amoxycillin/clavulanate accounted for the vast majority of pathogens isolated in early VAP. Suggestions that the distinction between early and late VAP should be abandoned emanate from the developed world where widespread antimicrobial use has led to a high level of bacterial resistance within the community. Extrapolating such data to the developing world is inappropriate and the use of broad spectrum antimicrobials in all patients will simply encourage resistant strains. Our data underscore the need for stewardship and surveillance in individual environments.

Bacterial resistance to even the broadest spectrum antimicrobials has become a global health emergency. ${ }^{21}$ Microbiological surveillance and antimicrobial stewardship are imperative if the efficacy of current antimicrobial agents is to be maintained while simultaneously providing adequate therapy. This policy advocates treatment for the most common, but not all, possible pathogens; ${ }^{22}$ and, by identifying the most prevalent bacterial flora in a specific health care environment, correct empiric therapy may be achieved over $90 \%$ of the time..$^{20}$ Surveillance must take into account a number of factors, amongst which is prior antimicrobial exposure which may alter the sensitivities of even community acquired infections. Most studies highlight exposure in other health care facilities as the most common threat. Recent hospitalisation to an acute care facility within the past 90 days with administration of intravenous antibiotics and residents in nursing homes or long-term care institutions are cited as high risk patients for bacterial resistance. ${ }^{23}$ Chronic care institutions are especially problematic due to frequent transfers between hospitals and institutions and the excessive use of broad spectrum antimicrobials. ${ }^{23}$ Cumulative exposure has also been documented as an independent risk factor for bacterial resistance but there are scant data, however, on the effect of a single course of antimicrobials for community acquired nonpneumonic infection and changes in aetiology and bacterial sensitivity patterns for early and late onset VAP. Our data show that antimicrobials targeting community acquired infections alter the normal flora isolated from the first episode of VAP. The reverse holds true in that organisms isolated in antimicrobial naïve patients are most commonly those from the community, even in later episodes of nosocomial sepsis.

\section{Conclusion}

Knowledge of prior antimicrobial exposure in a patient with early or late VAP will assist in determining the correct empiric antimicrobial choice with regard to targeting hospital or community acquired pathogens. In those who have had prior antimicrobial therapy for the pathology which required admission to ICU, early onset VAP will rarely, if ever, be due to normal community acquired pathogens. Antimicrobials prescribed for late onset VAP should, therefore, cover more broadly. Conversely, late onset VAP in the antimicrobial naïve patient will most frequently be due to community acquired flora and narrow spectrum antimicrobials against these pathogens may suffice. A continuous surveillance system monitoring pathogen resistance must be in place within a specific unit.

Authors contributions - YR conceived and designed the study. YR, DJJM, KSSH and KPM did the literature search. YR and DJJM prepared the data. YR and DJJM did the analysis. All authors interpreted data, wrote the manuscript, and approved the final version.
Funding - No funding has been received.

Transparency declarations - None to declare.

Acknowledgements - We acknowledge all contributions from the Trauma Intensive Care unit and Medical Microbiology laboratory staff at Inkosi Albert Luthuli Central Hospital.

\section{ORCID}

Koleka P Mlisana (D) http://orcid.org/0000-0002-8436-3268

\section{References}

1. Magill SS, Klompas M, Balk R, et al. Developing a new national approach to surveillance for ventilator-associated events. Am J Crit Care. 2013;22:469-73. doi:10.4037/ajcc2013893.

2. Bekaert M, Timsit JF, Vansteelandt $S$, et al. Attributable mortality of ventilator associated pneumonia: a reappraisal using causal analysis. Am J Resp Crit Care Med. 2011;184:1133-9. doi:10.1164/rccm.20110508670C.

3. Kollef $\mathrm{MH}$. Ventilator-associated complications, including infectionrelated complications. Crit Care Clin. 2013;29:33-50. doi:10.1016/j. ccc.2012.10.004.

4. American Thoracic Society. Infectious diseases society of america: guidelines for the management of adults with hospital-acquired ventilator-associated, and healthcare-associated pneumonia. Am J Resp Crit Care Med. 2005;171:388-416. doi:10.1164/rccm.200405644ST.

5. Kollef $\mathrm{MH}$. Inadequate Antimicrobial Treatment: An Important Determinant of Outcome for Hospitalized Patients. Clin Infect Dis 2000;31(Supplement 4):S131-8. doi:10.1086/314079.

6. Swanson JM, Wells DL. Empirical antibiotic therapy for ventilatorassociated pneumonia. Antibiotics. 2013;2:339-51. doi:10.339/ antibiotics2030339.

7. Lawrence $\mathrm{KL}$, Kollef $\mathrm{MH}$. Antimicrobial stewardship in the intensive care unit. advances and obstacles. Am J Resp Crit Care Med 2009;179:434-8. doi:10.1164/rccm.200809-1394CP

8. Gastmeier P, Sohr D, Geffers C, et al. Early- and late-onset pneumonia: is this still a useful classification? Antimicrob Agents and Chemother. 2009:53:2714-8. doi:10.1128/AAC.01070-08.

9. Bergallo C, Jasovich A, Teglia O, et al. Safety and efficacy of intravenous tigecycline in treatment of community-acquired pneumonia: results from a double-blind randomized phase 3 comparison study with levofloxacin. Diagn Microbiol Infect Dis. 2009;63:52-61. doi:10.1086/648720.

10. Jones RN. Microbial etiologies of hospital-acquired bacterial pneumonia and ventilator-associated bacterial pneumonia. Clin Infect Dis. 2010 Aug 1;51(S1):S81-7. doi:10.1086/653053.

11. Ramsamy Y, Muckart DJJ, Bruce JL, et al. Empirical antimicrobial therapy for probable $v$. directed therapy for possible ventilatorassociated pneumonia in critically injured patients. S Afr Med J. 2016;106(2):196-200. doi:10.7196/SAMJ.2016.v106i2.9870.

12. Khan R, Al-Dorzi A, Ahmed FW. The impact of implementing multifaceted interventions on the prevention of ventilator associated pneumonia. Am J Infect Control. 2016;44:320-6. https://doi.org/10.1016/j.ajic.2015.09.025

13. Clinical and Laboratory Standards Institute (CLSI) Performance standards for Antimicrobial Susceptibility Testing. Twenty-fourth Informational Supplement M100-S25. 2015:36-64.

14. Winn WC, Koneman EWW. Koneman's color atlas and textbook of diagnostic microbiology. Lippincott Williams \& Wilkins, ISBN 9780781730143, 2008. Available from: https://books.google.co.za/ books?id=4gWwsEiMwu8C

15. Kollef $\mathrm{MH}$. The prevention of ventilator-associated pneumonia. $\mathrm{N}$ Engl J Med. 1999;340:627-34. doi:10.1056/NEJM199902253400807.

16. Kollef MH. Epidemiology and risk factors for nosocomial pneumonia. Clin Chest Med. 1999;20:653-70. doi:10.1086/314079.

17. Craven DE, Steger KA. Ventilator-associated bacterial pneumonia: challenges in diagnosis, treatment, and prevention. New Horiz. 1998;6(Suppl):S30-45. 
18. Saroj G, Sangeetha KT, Vasudha CL. Microbial profile of early and late onset ventilator associated pneumonia in the intensive care unit of a tertiary care hospital in Bangalore, India. J Clin Diagn Res. 2013 Nov 11:2462-6.

19. Giard MA, Lepape A, Allaouchiche B, et al. Early- and late-onset ventilator-associated pneumonia acquired in the intensive care unit: comparison of risk factors. J Crit Care. 2008;23:27-33. doi:10.1016/j. jcrc.2007.08.005.

20. Ramsamy Y, Muckart DJJ, Han KSS. Microbiological surveillance and antimicrobial stewardship minimise the need for ultrabroadspectrum combination therapy for treatment of nosocomial infections in a trauma intensive care unit: An audit of an evidencebased empiric antimicrobial policy. S Afr Med J. 2013;103:371-6. doi:10.7196/SAMJ.6459.
21. World Health Organisation. Antibiotic Resistance Global Report on Surveillance. Geneva: WHO Press; 2014

22. Kollef $\mathrm{MH}$. Hospital-acquired pneumonia and de-escalation of antimicrobial treatment. Crit Care Med. 2001;29:1473-5. doi:10.1097/ 2F00003246-200107000-00029.

23. Borgatta B, Rello J. How to approach and treat VAP in ICU patients. BMC Infect Dis. 2014;14:867. Available from: http://www.biomedcentral. com/1471-2334/14/211. doi:10.1186/1471-2334-14-211.

24. Bonomo RA. Multiple antibiotic-resistant bacteria in long-term-care facilities: an emerging problem in the practice of infectious diseases. Clin Infect Dis. 2000;31:1414-22. doi:10.1086/317489.

Received: 21-10-2016 Accepted: 29-03-2017 